\title{
Spatially Adaptive Image Denoising using Undecimated Directionlet Transform
}

\author{
Sethunadh R \\ Vikram Sarabhai Space Centre \\ Indian Space Research Organisation (ISRO) \\ Trivandrum, Kerala, India
}

\author{
Tessamma Thomas \\ Department of Electronics \\ Cochin University of Science \& Technology \\ Cochin, Kerala, India
}

\begin{abstract}
In this paper a novel de-noising method based on directionlet transform and on sub band adaptive Bayesian threshold is presented. The denoising scheme used in wavelet domain has been extended to the directionlet domain to make the image features to concentrate on fewer coefficients so that more effective thresholding is possible. Here the directionality of the spatially segmented image is first computed using a parameter called directional variance for selecting the optimum direction for decomposing the image using undecimated directionlet transform. The decomposed images with directional energy are used for threshold computation using Bayes scheme. This threshold is then applied to the subbands except the LLL subband. The threshold corrected subbands with the unprocessed first sub-band are given as input to the inverse directionlet algorithm for getting the de-noised image. Experimental results show that the proposed method outperforms the standard wavelet-based denoising methods in terms of perceptual and numerical estimates.
\end{abstract}

\section{General Terms}

Image Denoising

\section{Keywords}

Undecimated directionlet transform, Multi resolution analysis, Directional variance, Image Denoising, Bayes threshold.

\section{INTRODUCTION}

An image is often corrupted by noise in its acquisition and transmission. The goal of image denoising is to remove noise while retaining the important signal features. There are linear and non-linear methods for image denoising. The linear processing methods include classical Wiener filtering. The non-linear techniques are mostly based on multi resolution analysis using wavelets $[1,2]$. The merit of denoising based on wavelet transform is multi scale frequency filtering characteristic, without using the statistical distribution of image. The classical wavelet based denoising method was proposed by Donoho and Johnstone [3]. It involves wavelet decomposition of the noisy image, classification of each wavelet coefficient as signal or noise and reconstruction of the image estimate using only signal coefficients. The simplest and common classification procedure is thresholding of wavelet coefficients. Here a wavelet coefficient is compared with a given threshold and is set to zero if its magnitude is less than the threshold; otherwise it is kept or modified based on hard or soft thresholding rules. Different approaches were proposed to estimate the proper threshold based on some optimisation criteria. The Bayesian threshold proposed by Chang et al [4] is based on an empirical observation in which the wavelet coefficients in each subband are modeled as independent and identically distributed random variables with Generalised Gaussian Distribution (GGD).

Wavelet coefficient thresholding scheme of de-noising is based on the idea that the energy of the signal to be defined concentrates on some of the wavelet coefficients, while the energy of noise spreads throughout all wavelet coefficients. Similarity between the basic wavelet and the signal to be defined plays a very important role, making it possible for the signal to concentrate on fewer coefficients..

Since the standard 2-D wavelet transform (WT) is a tensor product of 1-D WT, it has only three directions, viz. vertical, horizontal and diagonal. The 2-D WT is effective at approximating point singularities than line singularities like edges and boundaries due to isotropic scaling of its basis functions. Therefore a more effective basis for real-world images with edges and curves is required for making the signal to concentrate on fewer coefficients after transformation. Recently many multi-scale transforms with directional selectivity were developed for image representations. Some examples are curvelet [5], contourlet $[6,7]$ bandlet [8], shearlet [9] etc. Owing to the fact that multi-scale transforms with directivity provide image representations of high-energy concentration, the image denoising methods based on these transforms generally outperform WT based methods. However, these transforms often require oversampling, have higher complexity when compared to the standard WT, and require non-separable convolution and filter design. Also in some of these schemes the transform directions are not adaptive to the dominant directions and filtering is done in continuous domain making it difficult to use them on discrete images.

A special member of the emerging family of multi-scale geometric transforms is the directionlet transform (DT) which was proposed by V. Velisavljevi'c et al as an anisotropic, perfect reconstruction and critically sampled basis functions with directional vanishing moments along any two directions [10]. It retains the computational efficiency and the simplicity of 1-D processing and filter design from the standard separable 2-D WT. It has good approximation properties as compared to the approximation achieved by other over complete transform constructions and is superior to the performance of the standard separable 2-D WT while having the same complexity.

The directionlet transform is not shift - invariant due to the presence of down samplers and up-samplers in it. The undecimated directionlet transform which provides a shift invariant multidirectional image representation solves this problem. Here the Bayesian wavelet shrinkage scheme proposed by Chang et al [4] has been extended to the directionlet domain to better utilize the image specific directional features for image denoising.

The paper is organized as follows. In section 2, the theoretical concepts of directionlet transform and adaptive Bayes threshold are described. In section 3, the proposed directionally adaptive image denoising algorithm using Bayes 
shrinkage rule is discussed. The experimental results are presented in section 4 and the conclusions in section 5 .

\section{REVIEW OF BACKGROUND WORK}

Here a short review of the basic concepts of directionlet transform proposed by V. Velisavljevi'c et al [10] and adaptive Bayes threshold scheme proposed by S. G. Chang et al. [4] are given.

\subsection{Directionlet Transform}

The standard Wavelet Transform (WT) is an efficient tool for analysing one dimensional signal. However, for 2-D signals like images it is inefficient due to the spatial isotropy of its construction. In 2-D WT the number of 1-D transforms including filtering and sub-sampling operations along the horizontal and vertical directions is the same at each scale (Figure 1(a)). This isotropic transform cannot properly capture the anisotropic discontinuities present in images. This is because the directions of the transforms and discontinuities in images are not matched and the transforms fail to provide a compact representation of two dimensional signals. The standard WT considers only vertical and horizontal directions and number of filtering in both these directions is equal. Because of this, a separable 2D wavelet transform applied on natural images with edges in orientations other than horizontal and vertical, results in too many non-zero coefficients. Here the 1-D discontinuities like edges or contours of highly anisotropic objects cannot be properly captured by the isotropic WT. Many wavelets intersect the discontinuity and this leads to many large magnitude coefficients (see Figure $1(\mathrm{c}))$

In the anisotropic wavelet transform $\left(\operatorname{AWT}\left(n_{1}, n_{2}\right)\right)$, the number of transforms applied along the horizontal and vertical directions is unequal, that is there are $n_{1}$ horizontal and $n_{2}$ vertical transforms at a scale, where $n_{l}$ is not necessarily equal to $n_{2}$. The iteration process is continued in the low sub-band, like in the standard wavelet transform (Figure 1(b)). As opposed to the 3-band 2-D wavelet Transform, anisotropic wavelet transform produce three high-pass sub-bands per scale denoted as HL, LH and HH according to the order of low-pass and high-pass filtering in the two transform steps. The anisotropic ratio $\rho=n_{1} / n_{2}$ determines elongation of the basis functions of the AWT $\left(n_{1}, n_{2}\right)$. When $n_{1}=2, n_{2}=1$, the AWT $(2,1)$ produces eight bands viz. HHH, HHL, HLH, HLL, LHH, LHL, LLH, LLL as shown in Figure2. The skewed AWT can trace the discontinuity efficiently with fewer significant coefficients compared with standard WT (see Figure 1(d)).

The main problem with AWT is directional interaction. The lattice based transform can avoid the directional interaction. Here the discrete space is first partitioned using integer lattices before performing 1-D filtering along lines across the lattice. Any integer lattice $\Lambda$ is a sub-lattice of the cubic lattice $\mathrm{Z}^{2}$. Here the lattice $\Lambda$ can be represented by a non-unique generator matrix $\mathrm{M}_{\Lambda}$.

$\mathbf{M}_{\Lambda}=\left[\begin{array}{ll}a_{1} & b_{1} \\ a_{2} & b_{2}\end{array}\right]=\left[\begin{array}{l}d_{1} \\ d_{2}\end{array}\right]$

where, $\mathrm{a}_{1}, b_{1}, a_{2}, b_{2} \in Z$.

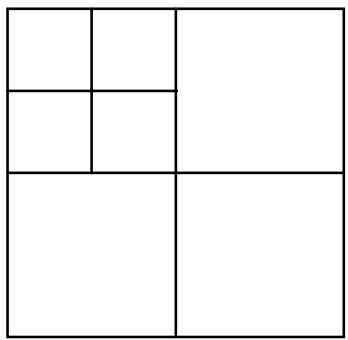

(a)

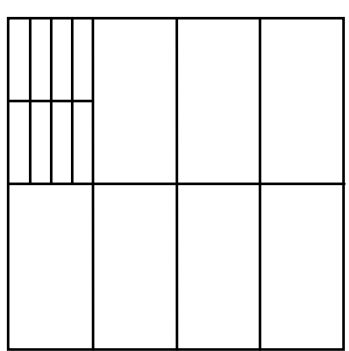

(c)

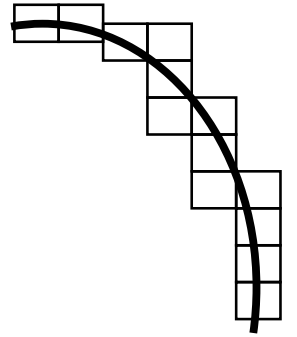

(b)

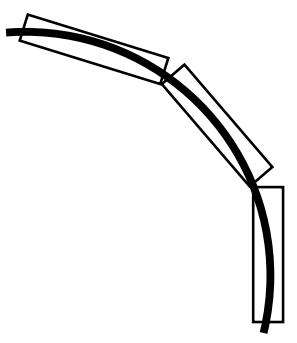

(d)

Figure 1. Frequency decomposition of (a) Standard 2-D Wavelet Transform (b) Anisotropic Wavelet Transform Basis functions of (c) 2-D Wavelet Transform (d) Anisotropic Wavelet Transform.

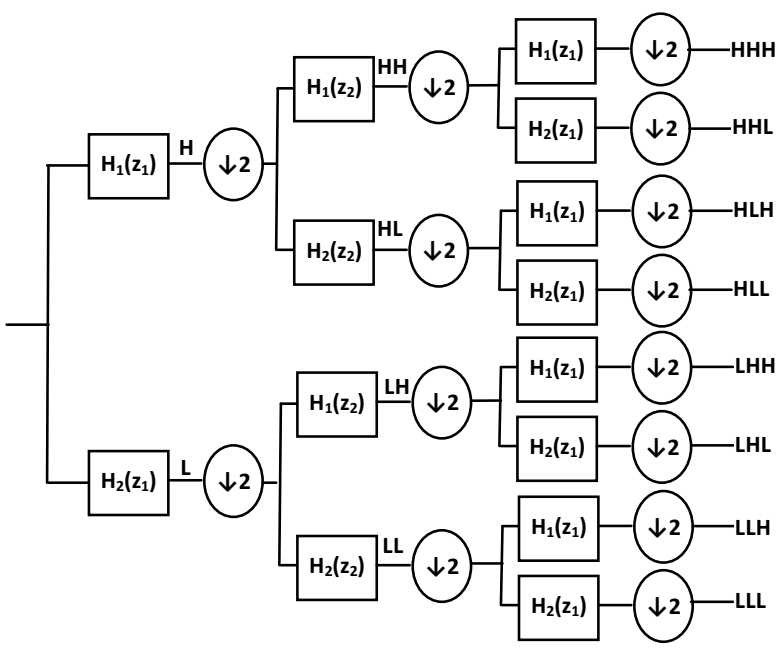

Figure 2. Filtering scheme for the AWT $(2,1)$, where one step of iteration is shown.

The linear combination of two linearly independent integer vectors $d_{l}$ and $d 2$ will form the points of the lattice $\Lambda$. The cubic lattice $Z^{2}$ can be partitioned into $\left|M_{\Lambda}\right|$ cosets of the lattice $\Lambda$. The filtering and sub sampling operations are applied on the pixels along the vector $d_{l}$ (transform direction) in each of the cosets separately. Since these operations are applied in each cosets separately, the pixels retained after this are clustered along the vector $d_{2}$ (alignment direction). This type of lattice based transform, which will avoid directional interaction, is called Skewed AWT, S-AWT $\left(\mathrm{M}_{\Lambda}, n_{l}, n_{2}\right)$. The basis functions of S-AWT are called directionlets, which can be effectively used for directional analysis of images. An example of construction of directionlets based on integer lattices is shown in Fig. 3 for pair of direction $\left(45^{\circ},-45^{\circ}\right)$. Here there are two cosets corresponding to the shift vectors $S_{0} \& S_{1}$. 

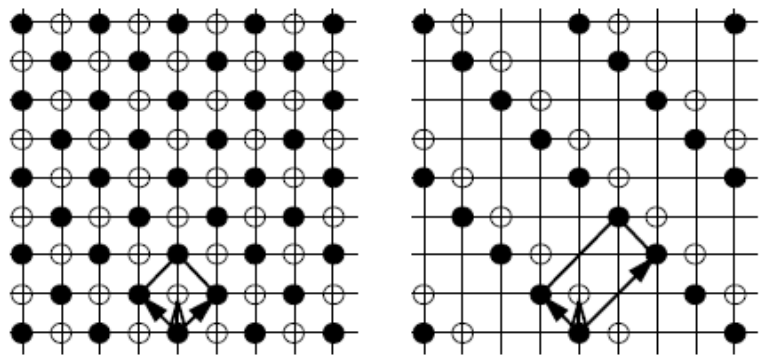

$$
M_{\Lambda}-\left[\begin{array}{cc}
1 & 1 \\
-1 & 1
\end{array}\right] \quad \bigcirc s_{1}-\left[\begin{array}{ll}
0 & 1
\end{array}\right]
$$$$
M_{\Lambda^{\prime}}-\left[\begin{array}{cc}
2 & 2 \\
-1 & 1
\end{array}\right]
$$

Figure 3. An example of construction of directionlets based on integer lattices for pair of directions $\left(45^{\circ},-45^{\circ}\right)$.

The directionlet transform is not shift invariant due to the presence of down sampling operation involved. The down sampling operation results in a time-variant translation and has difficulties preserving original image discontinuities in the directionlet domain. So shift invariance is a desirable property for image denoising applications. The Undecimated Directionlet Transform (UDT) can be constructed by avoiding the down sampling operation in the decomposition level. Thus the approximation and detail coefficients at each decomposition level are having the same length as the original signal. Here in the proposed scheme UDT was used for decomposition of the SAR image.

\subsection{Adaptive Bayes Threshold}

The Generalised Gaussian Distribution (GGD) has been used to model the coefficients in many wavelet based denoising applications. For most of the natural images, the WT coefficients from each subband (except LL) can be well described by the zero mean GGD. Based on this distribution many attempts were made to find out the best threshold for denoising applications The Bayes threshold based on GGD was first proposed by Chang et al [4] in the wavelet domain. The same scheme can be extended to the directionlet domain as DT is an extended version of WT. The threshold estimation problem in directionlet domain can be formulated as follows.

Suppose that the image $\mathrm{x}$ is polluted by independent and identically distributed white Gaussian noise $e$ with zero mean and $\sigma^{2}$ variance, the observed image is described as:

$$
y=x+v
$$

Since the directionlet transform is a linear operator, we can get the corresponding observation model in the directionlet domain as

$$
Y=X+V
$$

where $Y, X$ and $V$ are the directionlet transforms of $y, x$ and $v$ respectively.

\section{The GGD is}

$$
G G_{\sigma_{x}, \beta}(X)=C\left(\sigma_{x}, \beta\right) e^{-\left[\propto\left(\sigma_{x}, \beta\right)|x|\right]^{\beta}}
$$

Where,

$$
-\infty<x<\infty, \quad \sigma_{x}>0, \quad \beta>0
$$

$$
\begin{gathered}
\propto\left(\sigma_{x}, \beta\right)=\sigma_{x}^{-1}\left[\frac{\Gamma(3 / \beta)}{\Gamma(1 / \beta)}\right]^{1 / 2} \\
C\left(\sigma_{x}, \beta\right)=\frac{\beta . \propto\left(\sigma_{x}, \beta\right)}{2 \Gamma(1 / \beta)}
\end{gathered}
$$

and $\Gamma(1 / \beta)=\int_{0}^{\infty} e^{-u} u^{t-1} d u$ is the gamma function. The parameter $\sigma_{x}$ is the standard deviation and $\beta$ is the shape parameter. For a given set of parameter the objective is to find a soft threshold $T$ which minimises the Bayes risk $\mathrm{R}(T)$, which is defined as

$$
\mathrm{R}(T)=E(\overline{\mathrm{X}}-\mathrm{X})^{2}=E_{x} E_{Y / X}(\overline{\mathrm{X}}-\mathrm{X})^{2}
$$

The optimal threshold $T^{*}$ can be obtained by minimising the Bayes risk $\mathrm{Y}(T)$.

$$
T^{*}\left(\sigma_{x}, \beta\right)=\arg \min \mathrm{R}(T)
$$

This is a function of $\sigma_{x}$ and $\beta$. There is no closed form solution for $T^{*}\left(\sigma_{x}, \beta\right)$ for this chosen pair. Chang et al [4], proposed a numerical calculation to find its value.

$$
T_{B}\left(\sigma_{x}\right)=\frac{\sigma^{2}}{\sigma_{x}}
$$

\section{SPATIALLY ADAPTIVE IMAGE DENOISING}

The directionlet based denoising scheme proposed here is an extension of the wavelet based denoising techniques. The undecimated version of directionlet transform is used here to have better denoising performance. The directionlet has directional vanishing moments along two directions only. So for identifying the best pair of directions, directionlet transform has to be taken along multiple directions. This is computationally intensive and data expansive. Thus if the texture direction is known, the construction of directionlet can be limited to two dominant directions only. However the image can have different texture at different areas. This restriction implies a need for spatial segmentation of image and adaptation of the pair of directions in each segment based on the dominant direction of image. Here we propose a scheme by which the directional information of the spatially segmented image is first identified and the directionlet transform is computed for each spatial segment along two dominant directions. Bayesian subband adaptive thresholding is then applied to the decomposed image before reconstruction. Here we assume that the image is corrupted with independent and identically distributed (iid) Gaussian noise of zero mean and $\sigma^{2}$ variance. The resulting estimates will indeed consist of block artifacts as shown in figure 6. A simple procedure to reduce these artifacts is to apply a moving average filter in a small neighborhood of each block boundary. Here we have used the algorithm proposed in [11] for deblocking. This considerably reduces the block artifacts present in the processed image.

\subsection{Undecimated Directionlet Transform}

Unlike the discrete version of directionlet transform, which down samples the approximation coefficients and detail coefficients at each decomposition level, the Undecimated Directionlet Transform (UDT) does not incorporate the down sampling operations. Thus, the approximation coefficients and detail coefficients at each level are having the same length as the original signal. Denoising with the UDT is shift-invariant. 
The denoising result of the undecimated directionlet transform has a better balance between smoothness and accuracy than its discrete version. Oversampling is imposed in the same way as in the case of the undecimated wavelet transform, that is, by discarding the sub-samplers in the filter-banks of figure 2 . The filters used for DT are up sampled across scales. Thus, if the LP filter $H_{2}\left(Z_{1}\right)$ is used in the corresponding 1-D wavelet filter-bank, then the equivalent LP filter at the $j^{\text {th }}$ scale is $H_{2}\left(Z_{1}^{J-j}\right)$, where $j=1 \ldots J$, corresponds to the scale index sweeping from the finest to the coarsest scale. Such a construction results in a shift-invariant transform with a preserved number of coefficients in each subband.

\subsection{Directional variance}

Edge information in image has great influence on human visual effect. The information on the dominant direction of texture in an image can improve the precision of estimation of correct threshold for denoising. Thus it is important to identify the dominant direction of texture before thresholding. In this paper, the input image is first divided into spatial segments of smaller size. Then we construct a local texture-direction detector by measuring a parameter called directional variance to identify the pair of transform directions.

The directional variance, which measures the resulting residues when the signal is approximated with zero order polynomials along discrete lines in a given direction, was proposed by D. Jayachandra and A. Makur [12].

Given any rational slope $r$, the digital line $L(r, n)$ where $n \in \mathrm{Z}$, is defined as the set of pixels $(i, j)$ such that

$$
\begin{aligned}
& j=\lceil r i\rceil+n, \forall_{i} \in Z \text { for }|r| \leq 1 \text { or } \\
& i=\left\lceil\frac{j}{r}\right\rceil+n, \forall_{j} \in Z \quad \text { for }|r|>1 .
\end{aligned}
$$

For every rational slope $r$, the set of digital lines $L(r, n): n \in \mathrm{Z}$ completely partitions the $2 \mathrm{D}$ discrete space $Z^{2}$, meaning every pixel $(i, j) \in \mathrm{Z}^{2}$ is associated to only one of the digital lines with slope $r$.

As explained by D. Jayachandra and A. Makur [11], given the definition of digital line $L(r, n)$ with rational slope $r$, the directional variance for a given image segment $X$, along the lines with rational slope $r$, is defined as

$$
\operatorname{DirVar}(X, r)=\frac{1}{N \sum_{i=1}^{n} \sum_{j=1}^{k_{i}}\left(X_{j}-X_{L(r, i)}\right)^{2}}
$$

where, $X_{L(r, i)}$ is the mean of the digital line with slope $r$ and offset $i$, and $X_{j}$ is the pixel in the same line. $N$ is the total number of pixels in the segment $X, n$ is the total number of lines, and $k_{i}$ is the number of pixels in line $i$.

$\operatorname{DirVar}(X, r)$ measures the normalized sum of variances along each digital line with the given slope $r$ and hence is very sensitive to content directionality. DirVar measures spatial activity along the given direction. Here in the proposed method we have computed the directional variance along the rational directions $(i, j)=(1,0),(2,1),(1,1),(1,2),(0,1),(-1$, 1 ), which corresponds to $0^{\circ}, 30^{\circ}, 45^{\circ}, 60^{\circ}, 90^{\circ}$ and $-45^{\circ}$. This was carried out for each spatial segment of the image and the directions corresponding to the two minimum directional variances were identified and these were selected as the optimal pair of directions (transform direction and alignment direction) for computing the directionlet transform. For segments with no apparent dominant directions (where the directional variance for all the directions comes within 5\%), the pair $(0,90)$ is assigned by default to smooth the segments for the reason of simplicity of implementation of the directionlet.

\subsection{Bayes threshold computation in directionlet domain}

The directionlet coefficients of image show a strong nonGaussian statistical properties, whose probability density function can be modeled with the use of Generalized Gaussian Distribution. Thus the Bayesian threshold as given in equation (7) can be applied to the directionlet coefficients also. Zhang Wei et al. (2009), showed that the Bayesian threshold $T_{B}\left(\sigma_{x}\right)$ which is a function of GGD parameters $\sigma_{x}$ and $\beta$ is adaptive to different subband characteristics. Here the parameter $\beta$ doesn't explicitly enter into the equation. The noise variance $\sigma^{2}$ can be computed from the high frequency sub band coefficients at level1 $\left(\mathrm{HHH}_{1}\right)$ in the directionlet decomposition as:

$$
\sigma=\frac{\text { Median }\left(\left|K_{i j}\right|\right)}{0.6745}, \text { where } K_{i j} \in H H H_{1}
$$

The next parameter to be estimated is $\sigma_{X}$. From the image noise model as given in equation (3) and assuming that $\mathrm{X}$ and $\mathrm{V}$ are independent of each other,

$$
\sigma_{\mathrm{Y}}^{2}=\sigma_{\mathrm{X}}^{2}+\sigma^{2}
$$

where $\sigma_{\mathrm{Y}}^{2}$ is the variance of the noisy image. Since noisy image is modelled as zero mean, $\sigma_{\mathrm{y}}^{2}$ can be computed using the following empirical formula:-

$$
\sigma_{Y}^{2}=\frac{1}{n^{2}} \sum_{i, j=1}^{n} Y_{i j}^{2}
$$

Where $\mathrm{n} \times \mathrm{n}$ is the size of the sub band under consideration. $\sigma_{X}$ can now be computed as

$$
\sigma_{X}=\sqrt{\max \left(\sigma_{Y}^{2}-\sigma^{2}, 0\right)}
$$

From the computed values of $\sigma_{X}$ and $\sigma$ the threshold can be calculated using equation (7). The computed threshold is data driven and subband dependent.

\subsection{Thresholding functions}

Normally hard thresholding and soft thresholding functions are used for denoising process. Hard thresholding is "keep or kill" procedure and is more intuitively appealing but it introduces artifacts in the recovered images. The soft thresholding operation sets the coefficients smaller than the threshold to zero and shrinks the others toward zero. Soft thresholding is more efficient and it is also found to yield visually more pleasing images. Here in the proposed work the subband specific thresholds were applied to the coefficients using soft thresholding rule. The following illustration shows soft and hard thresholding operations. 


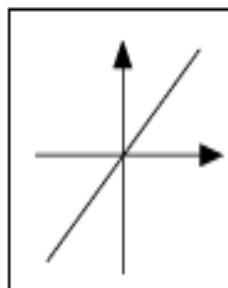

Source

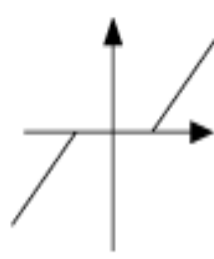

Solt Thresholding

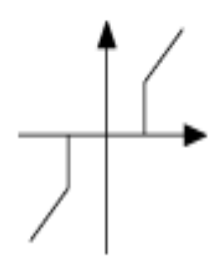

Hard Thresholding
Figure 4. Soft \& Hard thresholding operations

\subsection{Denoising algorithm}

The image denoising algorithm developed in the paper can be summarized as follows:-

Step 1: The noisy image is first divided into spatial segments of smaller size, say 64 by 64 .

Step 2: The DirVar along six directions $0^{\circ}, 30^{\circ}, 45^{\circ}, 60^{\circ}, 90^{\circ}$ and $-45^{\circ}$ are computed using equation (9) for each segment and the first two minimum DirVar directions are chosen as the pair of transform and alignment directions.

Step 3: The image segments are decomposed into sub-bands using directionlet transform along the chosen pair of directions.

Step 4: The noise variance is computed from the $\mathrm{HHH}_{1}$ subband using equation (10)

Step 5: For each subband, except the LLL subband, compute the Bayes threshold using the equation (7).

Step 6: Apply the computed threshold to the subbands using soft thresholding rule to estimate the best value for the noisefree coefficients.

Step 7: Reconstruct the image from the above processed subbands and the low-pass residual (LLL) to obtain the denoised image.

Step 8: Apply deblocking algorithm to remove the block artifacts.

\section{RESULTS AND DISCUSSIONS}

Standard grey images of size 512 x 512 were used for evaluating the performance of the developed algorithm. The test images were contaminated with zero mean white Gaussian noise with $\boldsymbol{\sigma}=10,15$, and 20 . The performance of the proposed method was compared with Bayeshrink in [4]. In our method we used the popular Haar wavelet for the decomposition of the image. The performance improvement was quantified in terms of Peak Signal to Noise Ratio (PSNR). PSNR was obtained by using the following formula:

$$
P S N R=10 \log _{10}\left(\frac{R^{2}}{M S E}\right)
$$

where $R$ is the maximum fluctuation in the denoised image. For an 8 bit gray scale image the value of $\mathrm{R}$ is 255 . MSE is representing the Mean Square Error between the denoised image $I_{d e n}$ and the original image (before adding noise) $I_{\text {org }}$, which was computed using the following equation:

$$
M S E=\frac{\sum_{i, j}\left(I_{d e n}(i, j)-I_{\text {org }}(i, j)\right)^{2}}{M x N}
$$

where $\mathrm{M}$ and $\mathrm{N}$ are the size of the images.

The directions estimated by minimizing the directional variance for the Lena image is shown in Figure 5. Here the image was segmented into small patches of size $32 \times 32$ and the directional variance was computed along eight different directions. It is evident from this figure that the directional variance has correctly identified the directions that are close to the content directionality of the image. The blocking effect due to the block processing as explained earlier is shown in Figure 6 for Lena image.

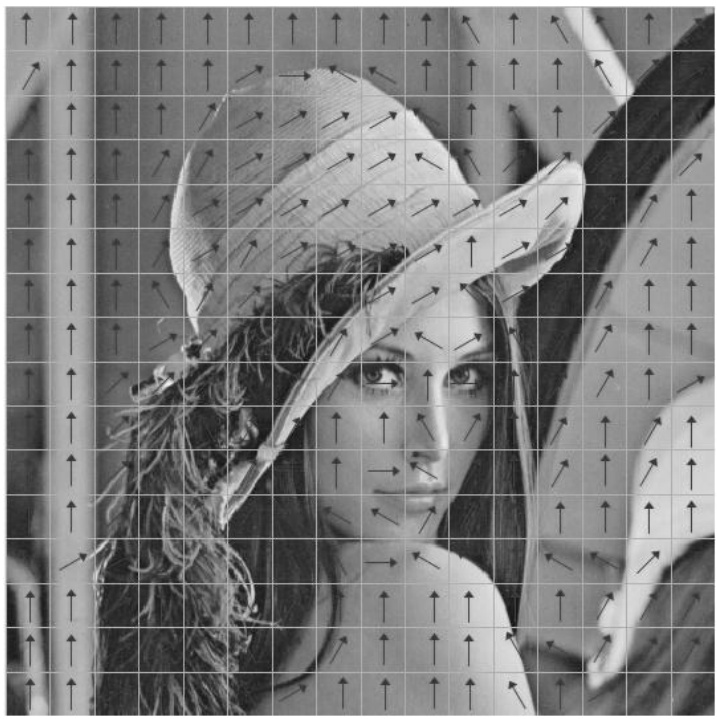

Figure 5: Directions estimated by minimizing Directional Variance for Lena image.

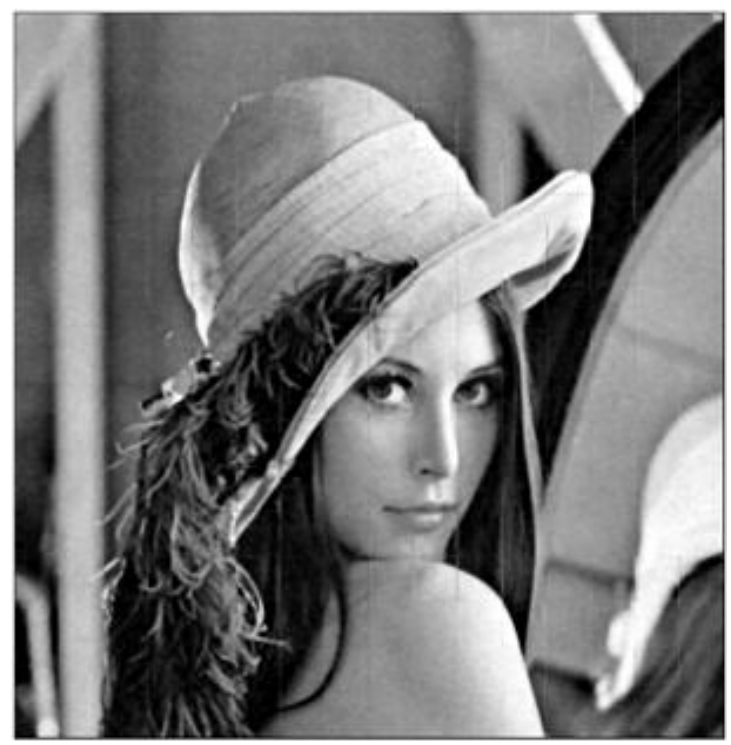

Figure 6: The denoised images of Lena before applying the deblocking algorithm. The sharp artificial edges are visible at the segment boarders.

The PSNR comparison of Lena, Boat and Barbara images is given in Table 1 and a visual comparison of Lena and Barbara images in Figure 7 for $\sigma=20$. The PSNR values provided 
are the average of 10 different noise realisations for each standard deviation. The proposed method outperforms the other ones for both the test images. Also the visual quality of the denoised images is evidently better because of sharper edges and texture. The PSNR difference for Barbara image is more due to the fact that it has more high frequency content and so more directional features. It is evident from the result that the DT has effectively captured the directional features in Barbara image.
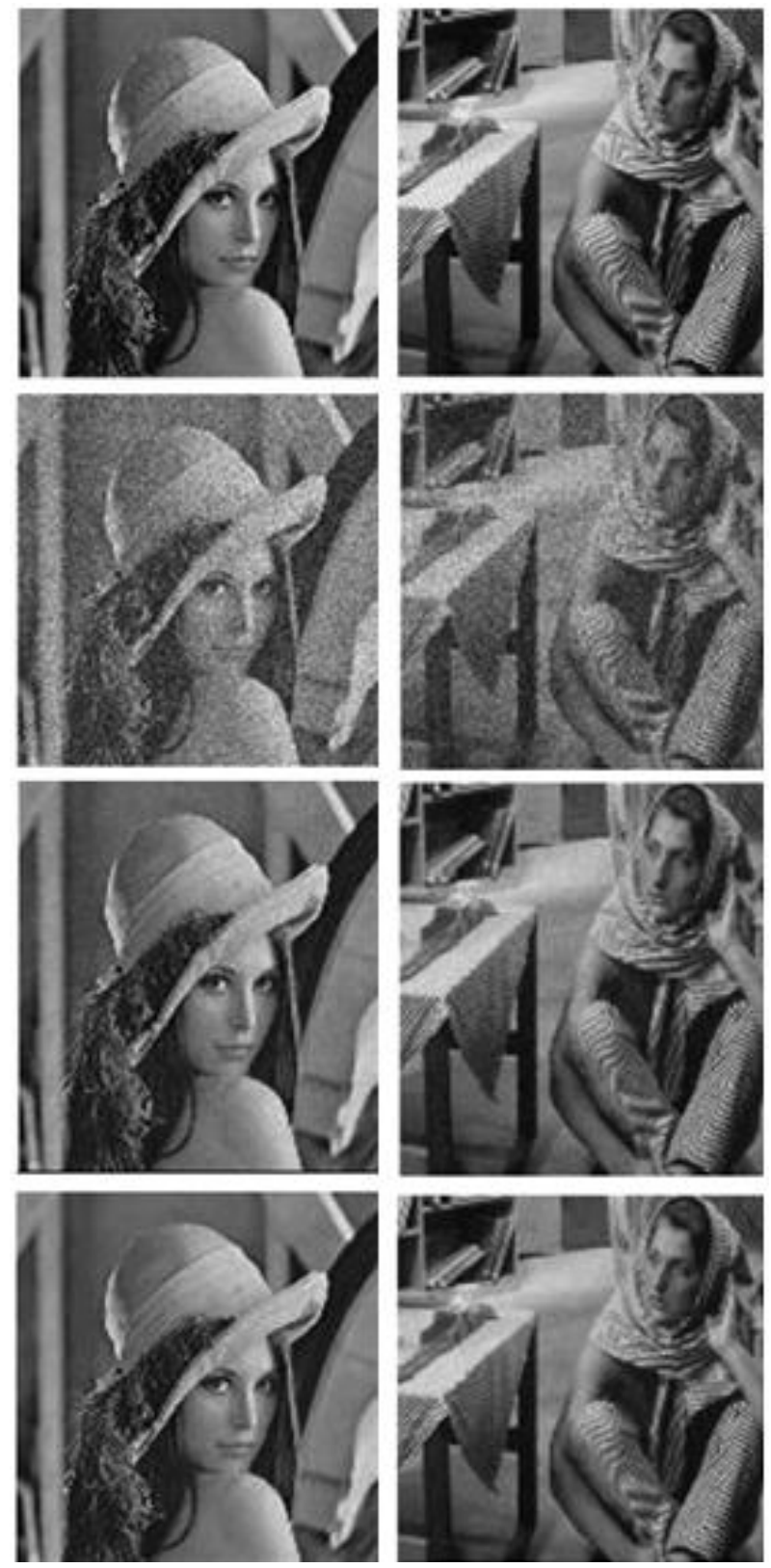

Figure.7. Image denoising using the new method for Lena and Barbara images. These images are presented in the order from top to bottom: Noise free image, noisy image with $\sigma=20$, denoised image using wavelet, denoised image using directionlet.
Table.1. PSNR (dB)* values for the different denoising algorithms of Lena \& Barbara $(512 \times 512)$ images

\begin{tabular}{|l|l|c|c|c|}
\hline \multirow{2}{*}{ Image } & \multirow{2}{*}{ Method } & \multicolumn{3}{|c|}{$\boldsymbol{\sigma}$} \\
\cline { 3 - 5 } & & 10 & 15 & 20 \\
\hline \multirow{3}{*}{ Lena } & Noisy Image & 28.15 & 24.63 & 22.11 \\
\cline { 2 - 5 } & BayesShrink & 33.31 & 31.39 & 30.14 \\
\cline { 2 - 5 } & Directionlet & $\mathbf{3 4 . 3 8}$ & $\mathbf{3 2 . 4 8}$ & $\mathbf{3 1 . 2 0}$ \\
\hline \multirow{3}{*}{ Barbara } & Noisy Image & 28.15 & 24.63 & 22.11 \\
\cline { 2 - 5 } & BayesShrink & 31.80 & 29.87 & 28.48 \\
\cline { 2 - 5 } & Directionlet & $\mathbf{3 2 . 4 6}$ & $\mathbf{3 0 . 6 1}$ & $\mathbf{2 9 . 1 9}$ \\
\cline { 2 - 5 } & Noisy Image & 28.15 & 24.64 & 22.11 \\
\cline { 2 - 5 } & BayesShrink & 30.85 & 28.50 & 27.13 \\
\hline & Directionlet & $\mathbf{3 2 . 2 9}$ & $\mathbf{3 0 . 1 1}$ & $\mathbf{2 8 . 4 5}$ \\
\hline
\end{tabular}

* Average of ten readings

\section{CONCLUSIONS}

The paper has proposed a new image denoising technique based on skewed anisotropic wavelet transform called directionlets. The new method suitably adjusts the transform directions based on dominant directions of each segment of image and successfully captures the oriented features. Here the dominant directions of the segmented image are first identified by computing the directional variance and those were selected as the suitable transform directions for taking the directionlet transform. The method is more computationally efficient than the method which takes the directionlet transform along multiple directions to identify the dominant directions. The Bayes Shrink method is used for setting subband adaptive thresholds to the decomposed image. The proposed technique has been tested on well-known benchmark images, where their PSNR and visual results show the superiority of the proposed technique over the conventional image denoising techniques. The performance improvement is basically due to the adaptation of the transform directions along the dominant directions of the spatially segmented image.

\section{REFERENCES}

[1] Mallat S, Hwang W L, "Singularity detection and processing with wavelets", IEEE Trans Inform Theory, Vol. 38, No. 2, pp. 617-643, 1992.

[2] Xu Y S, Weaver J B, Heal YD M, et al. "Wavelet transform domain filters: a spatially selective noise filtration technique", IEEE Trans Image Processing, Vol. 3, No. 6, pp. 747-758, 1994

[3] D. L. Donoho and I. M. Johnstone, "Adapting to unknown smoothness via wavelet shrinkage," J. Amer. Statist. Assoc., vol. 90, pp. 1200-1224,1995

[4] S. G. Chang, B. Yu, and M. Vetterli, "Adaptive wavelet thresholding for image denoising and compression," IEEE Trans. Image Processing, vol.9, pp. 1532-1546, 2000.

[5] Strack J.L., Candes E.J., Donoho D.L.: 'The curvelet transform for image denoising', IEEE Trans. Image Process., 2000, 11, (6), pp. 670-684 
[6] Do M.N., Vetterli M.: 'The contourlet transforms: an efficient directional multiresolution image representation', IEEE Trans. Image Process., 2005, 14, (12), pp. 2091-2106

[7] Eslami R., Radha H.: 'Translation-invariant contourlet transform and its application to image denoising', IEEE Trans. Image Process., 2006, 15, (11), pp. 3362-3374

[8] E. L. Pennec and S. Mallat, "Sparse geometrical image representations with bandelets,". IEEE Trans. Image Processing, Vol. 14, Apr. 2005, pp. 423-438.

[9] G. Easley, D. Labate, and W. Lim, "Sparse directional image representations using the discrete Shearlet transform," Appl. Comput. Harmon. Anal., vol. 25, no. 1, Jan. 2008, pp. 25-46.

[10] Vladan Velisavljevic ,Baltasar Beferull-Lozano ,Martin Vetterly and Pier Luigi Dragotti "Directionlets: Anisotropic Multi directional Representation with Separable Filtering", IEEE Transactions on Image processing, Vol 15 Issue 7, pp.1916-1933, 2006

[11] Z. Xiong, M. T. Orchard, and Y.Q. Zhang, "A deblocking algorithm for JPEG compressed images using overcomplete wavelet representations," IEEE Trans. Circuits Syst. Vid. Tech., vol. 7, no. 2, pp. 433-437, Apr. 1997.

[12] D. Jayachandra and A. Makur "Directional Variance: A Measure to Find the Directionality in a Given Image
Segment," IEEE International Symposium on Circuits And Systems, pp. 1551-1554, 2010.

\section{AUTHOR BIOGRAPHY}

Sethunadh $\mathbf{R}$ received his B.Tech in electronics \& communication from University of Kerala and M.Tech in digital electronics with first rank from Cochin University of Science and Technology, Cochin-22, India. He started his career in airworthiness certification at Indian Defence Research and Development Organisation (DRDO). Later he joined Vikram Sarabhai Space Centre, Indian Space Research Organisation (ISRO) and has been engaged in launch vehicle development program. He is also pursuing his $\mathrm{Ph} . \mathrm{D}$ at Department of Electronics, Cochin University of Science and Technology, India. His research interest includes image processing, computer vision, signal processing, test automation etc.

Dr.Tessamma Thomas received her M.Tech. and Ph.D from Cochin University of Science and Technology, Cochin-22, India. At present she is working as Professor in the Department of Electronics, Cochin University of Science and Technology. She has to her credit more than hundred research papers, in various research fields, published in International and National journals and conferences. Her areas of interest include digital signal and image processing, bio medical image processing, super resolution, content based image retrieval, genomic signal processing, etc. 